\title{
Pessoas com deficiência na atenção primária: discurso e prática de profissionais em um centro de saúde-escola
}

Marilia Bense Othero' Ana Sílvia Whitaker Dalmaso ${ }^{2}$

A atenção primária é um campo importante para o desenvolvimento de práticas de atenção à saúde da pessoa com deficiência, particularmente naquilo que tange à circulação e participação sociais, sob a ótica de inclusão e dos direitos de cidadania. Porém, este é um tema ainda pouco desenvolvido nas unidades básicas de saúde. A pesquisa ${ }^{3}$ aqui apresentada tem como objetivo conhecer as representações dos profissionais de um centro de saúde-escola da cidade de São Paulo sobre a deficiência e sobre o papel da atenção primária diante das necessidades desta população.

Na sociedade e na cultura, os pertencimentos das pessoas são múltiplos: gênero, etnia, condição econômica, religião, identidade cultural, entre tantos outros, que influenciam nos modos de ser e estar no mundo. Entretanto, o encontro com a diversidade é permeado por estigmas, estereótipos e desigualdades, que são construídos subjetiva, histórica, social e culturalmente. De um lado, encontram-se as desigualdades sociais, cujas raízes estão no próprio processo de construção da sociedade. De outro, estão os aspectos individuais de percepção das diferenças, relacionados às experiências pessoais, aos valores éticos e morais, e à história de vida. Estes processos intrinsecamente relacionados são constantes, uma vez que se constroem mútua e continuadamente.

Ao longo da história, as pessoas com deficiência foram consideradas ora amaldiçoadas, ora seres semidivinos, mas sempre excluídas do contexto social e objeto de caridade da comunidade (Pessoti apud Ghirardi, 1999). O imaginário e as representações sociais da deficiência contribuem para que a atenção à saúde desta população torne-se ainda mais complexa.

Segundo Vaitsman (2002), há uma negação da dignidade das pessoas diferentes, erguendo-se estereótipos e se vinculando uma qualidade negativa à diferença; com isso, produz-se segregação social e simbólica, dificultando a formação de processos sociais mais inclusivos.

Os profissionais de saúde são parte deste meio sociocultural, que ainda segrega as pessoas com deficiência e, portanto, as práticas em saúde são construídas a partir da relação socio-histórica-cultural com a questão. Para além da compreensão de suas necessidades - seja a partir de modelos médicos ou sociais, como aponta Ghirardi (1999) - perpassam os estigmas, a relação com a diversidade, as desigualdades sociais. Assim, é preciso conhecer a representação de profissionais

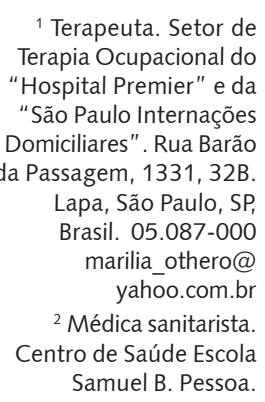

\footnotetext{
${ }^{3}$ Esta pesquisa compõe a monografia da primeira autora deste texto, elaborada para a conclusão do curso de aprimoramento em Saúde Coletiva, pela Faculdade de Medicina da Universidade de
} São Paulo. 
acerca deste fenômeno, como forma de tornar a prática em saúde aberta à crítica e atenta às reais necessidades das pessoas atendidas, construindo o que Schraiber e Mendes-Gonçalves (2000) nomeiam de contexto instaurador de necessidades.

Segundo Magnani (1986), a representação é tomada usualmente como uma imagem mental da realidade, composta pelas experiências individuais decorrentes da vida cotidiana do ator, envolvendo: a família, a vizinhança, o bairro, a categoria profissional, a vinculação política e a classe social, entre outros aspectos. Esta concepção remete à ênfase no discurso, envolvendo complexos processos de análise, que deixam, em segundo plano, aspectos extradiscursivos.

No trabalho de Malinowski com os baloma de Kiriwina, temos a descrição não só do que os indivíduos contam, mas do que fazem: "[...] as idéias e crenças não existem apenas nas opiniões conscientes e formuladas dos membros da comunidade; estão incorporadas em instituições sociais e estampadas nas condutas dos aborígenes e deverão ser extraídas, por assim dizer, de ambas as fontes" (Malinowski, 1974, p.315).

Aqui foi adotada a noção de representação baseada na concepção de Malinowski, ou seja, como discurso e prática que, incorporados no serviço, são construídos mutuamente, de maneira dialética. Assim, discurso e prática são aspectos que não se sobrepõem necessariamente e nem sempre se opõem, mas sim são complementares para a compreensão da realidade (Magnani, 1986).

Com sua prática e seu discurso, os profissionais de um serviço de saúde contribuem para a produção e reprodução do conhecimento e do significado da deficiência na sociedade e nos cuidados de saúde.

\section{Direito à saúde e pessoas com deficiência}

De acordo com a apresentação do Programa de Atenção à Saúde da Pessoa com Deficiência no Sistema Único de Saúde (Brasil, 1993), antes da criação do Sistema Único de Saúde (SUS) em 1988, a assistência às pessoas com deficiência, na saúde pública, era limitada somente à área de prevenção de doenças infectocontagiosas. A reabilitação era entendida como nível terciário da atenção, sob a responsabilidade de instituições filantrópicas e de associações beneficentes; as políticas públicas funcionavam no modelo de repasse de recursos para a filantropia. Segundo este documento, o panorama era de uma assistência muito precária: recursos insuficientes, serviços concentrados em regiões de maior relevância econômica, modelo assistencial com baixa cobertura, pouca resolutividade e inadequação à realidade sociocultural da população.

No contexto de reformas do final da década de 1970 e início da década de 1980, surgiram os movimentos sociais das pessoas com deficiência (que incluíam as pessoas, seus familiares e os profissionais da reabilitação), que tiveram importância fundamental na reivindicação de direitos sociais e igualdade de oportunidades (Rocha, 2006). Segundo a autora, estes movimentos associaram suas "bandeiras de luta" às demais existentes na sociedade brasileira, ou seja: lutas por direitos sociais, como serviços de saúde, por escolas, pelo fim da discriminação social e jurídica, pelo transporte, entre outras.

Com a pressão dessas mobilizações, o Estado passou a incorporar a temática da saúde da pessoa com deficiência como uma preocupação, propondo programas e políticas nacionais voltados para esta população. Em meados da década de 1980, a Secretaria de Estado da Saúde de São Paulo implantou o "Sistema de Atendimento em Saúde e Reabilitação para Pessoas Deficientes". No âmbito federal, em 1986, a criação da Coordenadoria Nacional para Integração da Pessoa Portadora de Deficiência (CORDE) foi uma referência na conquista dos direitos civis e sociais (Rocha, 2006).

$\mathrm{Na}$ área da saúde, todo o processo de mudanças sociais culminou na criação do SUS, sendo a saúde um direito de todos e um dever do Estado. Já como parte do SUS, há marcos importantes, elencados no Manual de Legislação em Saúde da Pessoa com Deficiência, publicado pelo Ministério da Saúde (Brasil, 2006): "Programa de Atenção à Saúde da Pessoa Portadora de Deficiência", de 1991; "Atenção à Pessoa Portadora de Deficiência no Sistema Único de Saúde - Planejamento e Organização de Serviços", de 1993; "Política de Integração da Pessoa Portadora de Deficiência", de 1999; "Política Nacional de Saúde da Pessoa com Deficiência", de 2002; além de portarias que foram criadas para a regulamentação das diretrizes e práticas. 


\section{Práticas em Atenção Primária}

Segundo Almeida, Tissi e Oliver (2000), a Organização Mundial de Saúde (OMS) prevê que $10 \%$ da população mundial apresentem algum tipo de deficiência. Neste grupo, cerca de 1,5\% são pessoas que se beneficiariam dos serviços de reabilitação; entretanto, estima-se que se consiga prover algum tipo de atendimento a apenas $2 \%$ das pessoas com deficiência que necessitem destes cuidados. É importante ressaltar que, como indivíduos, as pessoas com deficiência têm outras necessidades em saúde além da reabilitação, e, mesmo sob este aspecto, não costumam ser atendidas pelo atual sistema de saúde.

As autoras completam que, muitas vezes, a principal necessidade dessas pessoas não está na gravidade orgânica ou nos comprometimentos funcionais, mas sim em processos sociais extremamente complexos, que condicionam desigualdades sociais entre os cidadãos. Torna-se, portanto, imprescindível que os serviços se organizem para responder a estas demandas.

A atenção primária representa o espaço institucional específico para ações que não estão relacionadas somente à esfera biológica das patologias, além de permitir uma ampliação da cobertura assistencial. Neste campo de atenção, abre-se a possibilidade de atuação no chamado paradigma social (Ghirardi, 1999), que compreende e age sobre o fenômeno da deficiência para além do aspecto orgânico individual, incluindo as trocas sociais e a autonomia, e compreendendo a relação da pessoa com deficiência com o contexto histórico-social e com as dimensões subjetivas.

Adota-se o conceito de atenção primária como aquela que envolve baixa densidade tecnológica-material, mas que implica grande complexidade assistencial. Schraiber e Mendes-Gonçalves (2000) elencam três eixos principais:

- É uma forma de organização do trabalho, com suas complexidades particulares, que não deve reduzir as necessidades aos processos fisiopatológicos;

- Revaloriza a busca por uma assistência integral, isto é, não como uma somatória de atos especializados, sendo a unidade básica de saúde o local adequado para esta assistência global;

- Incorpora a dimensão subjetiva das práticas, tanto do profissional quanto do usuário, não havendo uma cisão entre o humano e o técnico-científico.

A partir de referências da Reabilitação Baseada na Comunidade (RBC) ${ }^{4}$, Oliver et al. (1999) propõem que um dos principais objetivos das ações em atenção

${ }^{1}$ RBC - Reabilitação Baseada na Comunidade: proposta assistencial para os serviços de atenção primária existentes no Sistema Único de Saúde. Foi criada pela OMS, no final da década de 1970 como uma contraposição à organização de serviços de reabilitação baseados no paradigma médico. Foi proposta como um modelo de ampliação da cobertura assistencial e para o combate à segregação e ao internamento das pessoas com deficiência (Oliver et al., 1999). primária, com relação à saúde de pessoas com deficiência, é dar-lhes visibilidade social, valorizando-se os processos e recursos locais para a construção da inclusão; parte-se do entendimento de que as pessoas são sujeitos com saberes, valores e história, pertencentes a um contexto sociocultural, e que podem agir sobre ele. Oliver et al. (2004) trazem outros eixos: a garantia de direitos, a construção de redes de apoio social e a criação de oportunidades de ampliação da sociabilidade. Almeida, Tissi e Oliver (2000) ressaltam, ainda, a importância de se atuar na construção de trocas sociais, tendo como horizonte ético a igualdade e o respeito à diferença.

\section{A pesquisa e seu desenvolvimento}

O estudo foi feito no Centro de Saúde-Escola (CSE) "Samuel B. Pessoa", unidade básica de saúde da zona oeste da cidade de São Paulo (bairro do Butantã), vinculado à Universidade de São Paulo como unidade docente-assistencial da Faculdade de Medicina. 
Para atender a uma população de cerca de 49 mil pessoas - segundo estimativa da Secretaria Municipal de Planejamento Urbano (Prefeitura de São Paulo, 2008) - o serviço conta com sete setores assistenciais principais: saúde da criança e do adulto, saúde mental, procedimentos de enfermagem (vacinação e curativos), farmácia, fonoaudiologia, saúde bucal, além dos setores de apoio (vigilância à saúde, estatística, gerência e administração). No funcionamento da unidade, existem programas voltados a grupos populacionais e problemáticas específicas: programa de saúde do adolescente, programa do envelhecimento, programa de saúde da mulher e do homem, programa de atenção primária domiciliar, programa de saúde mental, entre outros. Há também o Programa de Saúde da Família, para uma área específica (Jardim São Remo), na qual agentes comunitários de saúde desenvolvem ações de cadastramento das famílias residentes, visitas domiciliares e atividades comunitárias, integradas aos diversos setores do serviço.

A pesquisa foi realizada em três etapas, no período de outubro de 2005 a janeiro de 2006. A primeira caracterizou-se por um levantamento bibliográfico na base de dados Literatura LatinoAmericana e do Caribe (LILACS), sendo importante ressaltar que existe pouca bibliografia disponível sobre o tema.

Na segunda etapa, foram colhidos os dados primários do estudo: entrevistas com profissionais do centro de saúde e levantamento de registros em prontuários de pessoas com deficiência atendidas pelo serviço, de acordo com os preceitos de ética em pesquisa. Esta metodologia foi escolhida por permitir a produção de informações sobre o discurso dos profissionais (por meio das entrevistas) e sobre a prática dos mesmos (por intermédio da análise de prontuários), com base no conceito de representação adotado.

As entrevistas realizadas foram do tipo semiestruturada (roteiro previamente estabelecido), com seis profissionais tomados como "representantes-chave" do serviço: dois agentes comunitários de saúde, dois profissionais da equipe de enfermagem e dois médicos. A escolha de diferentes categorias profissionais teve como objetivo possibilitar uma melhor apreensão da representação da deficiência, uma vez que esta se conforma de acordo com o trabalho de cada um, também definido como o lugar do qual o sujeito fala e no qual atua.

Para análise dos prontuários, foram feitos estudos de caso de quatro prontuários de pessoas com deficiência que utilizaram o centro de saúde, escolhidos por meio de indicação de profissionais da unidade. Eram pacientes de idades variadas (três adultos e uma criança), sendo três do sexo feminino; todos receberam um primeiro atendimento na unidade, e posteriormente foram atendidos pelo programa de atenção domiciliar.

Os usuários atendidos na unidade têm um prontuário individual, e, para os moradores do Jardim São Remo, há um prontuário da família, no qual estão contidas as informações sobre o domicílio, cadastro individual dos moradores e registros das visitas realizadas pelos agentes comunitários. Nos estudos de casos, foram analisados ambos os tipos de registro.

Elaborou-se previamente um roteiro de leitura, a fim de se caracterizarem: o uso do serviço, as demandas e necessidades apontadas, as condutas e os encaminhamentos feitos pelos profissionais que os atenderam. O roteiro era composto por duas categorias principais: "focos da atenção", relacionada à assistência oferecida pelo serviço de saúde; e "organização dos cuidados", referindo-se à rede de cuidados da pessoa com deficiência e aos percursos realizados no sistema de saúde pelo paciente e sua família. Para ilustração, apresentamos, na Tabela 1, as informações colhidas dos registros em prontuário de um dos casos estudados.

Tabela 1. Informações sobre focos de atenção e organização do cuidado conforme registros em prontuário de usuário do CSE.

Caso B. 69 anos, sexo feminino, casada, natural de São Paulo. Mora com o esposo; a filha e sua família moram no mesmo terreno. Sofreu Acidente Vascular Cerebral (AVC) em 1994, com sequelas, e, em 2004, sofreu amputação em membro inferior esquerdo; profissionais que a atendem levantam hipótese de quadro demencial. Seu primeiro atendimento no serviço foi em 1997, em grupo de orientação para hipertensos; fez uso do serviço em consultas de seguimento no "Programa de Envelhecimento" e em pronto-atendimentos, com demandas relacionadas a diabetes e hipertensão. Em 2003, iniciou seguimento no Programa de Atenção Primária Domiciliar (APD), solicitado por enfermeira da unidade, mantendo-se em acompanhamento domiciliar até o momento. 


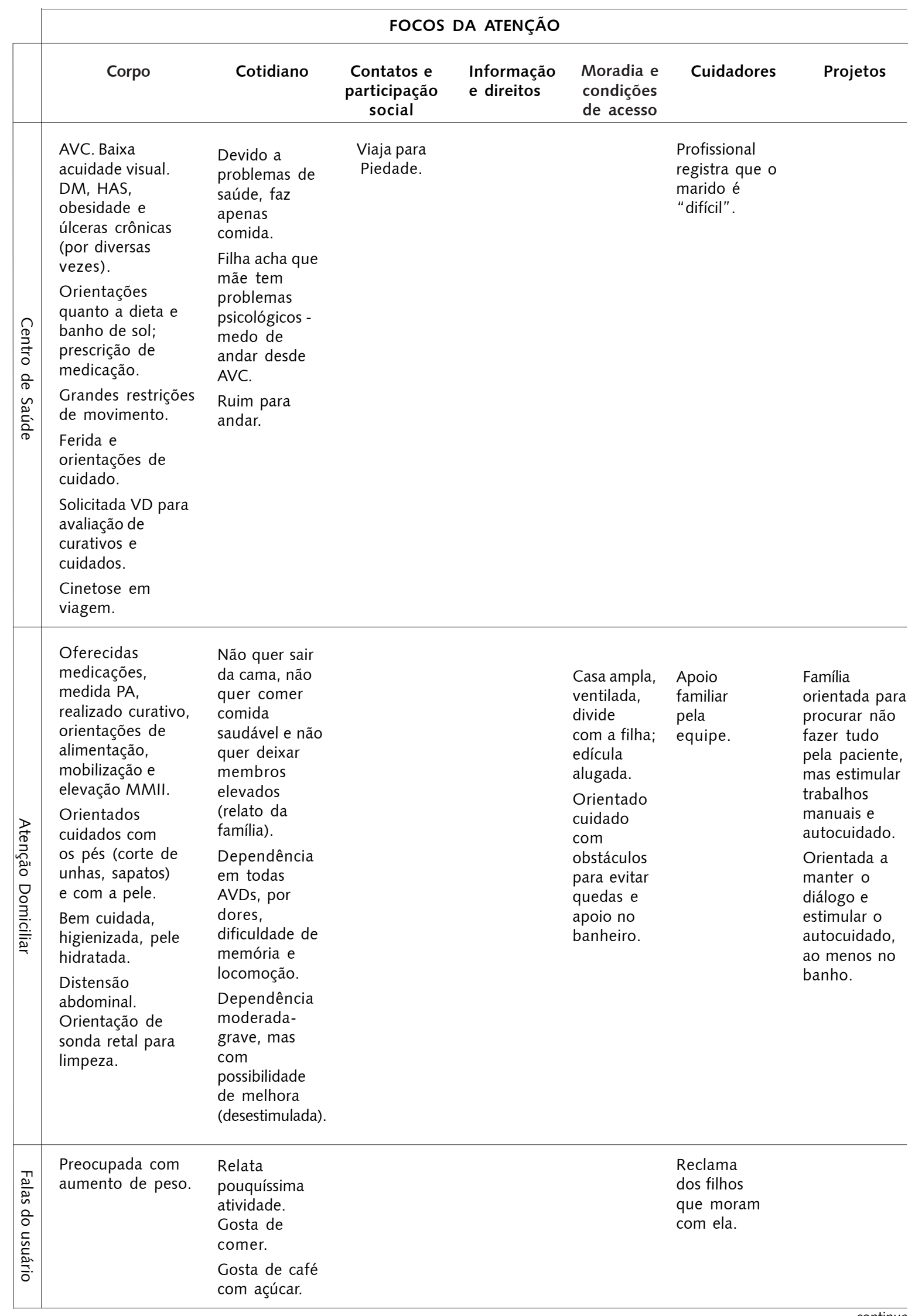


Tabela 1. continuação

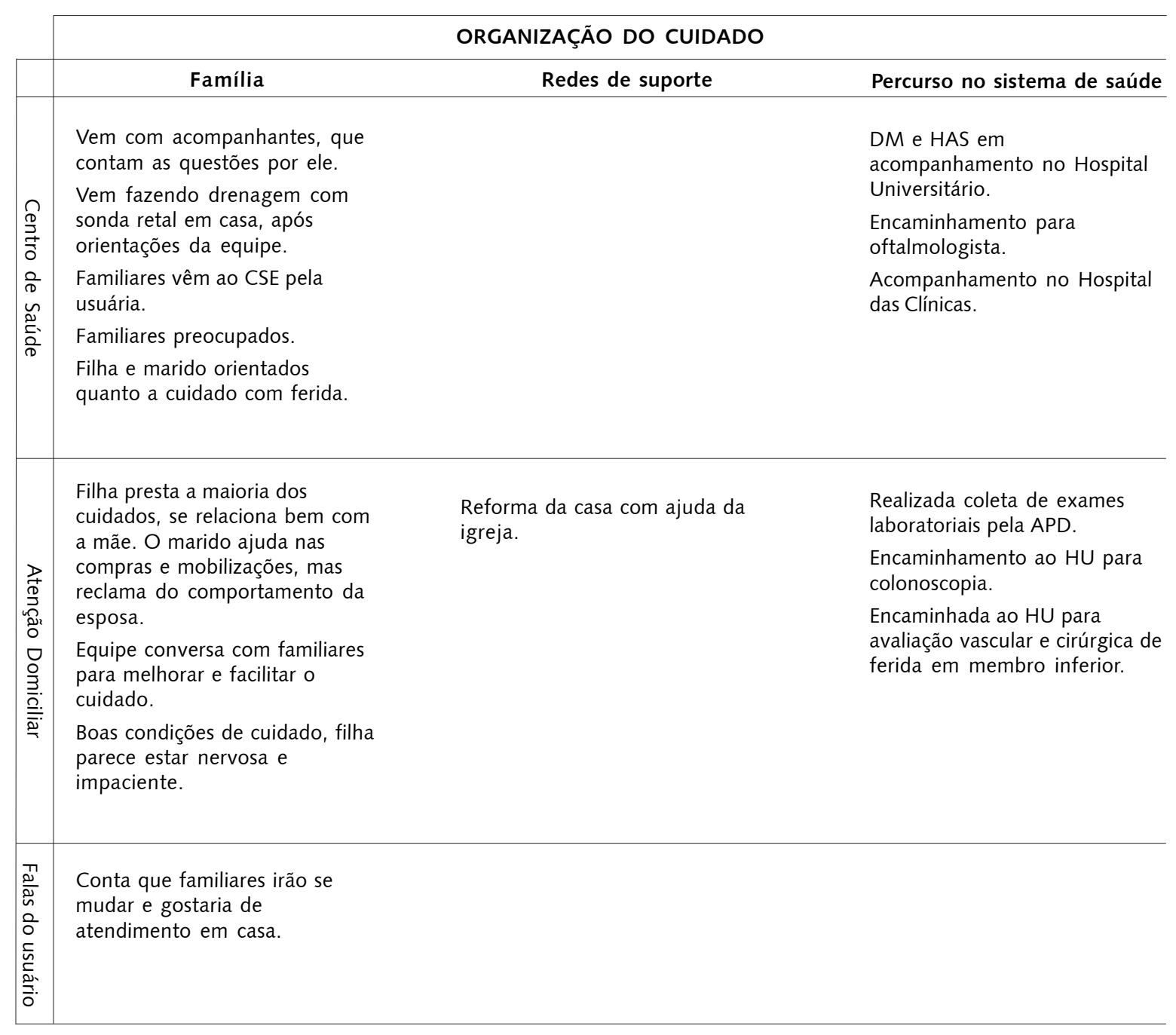

Por fim, em uma terceira etapa, foi feita a síntese dos resultados obtidos, com base na criação de categorias de análise dos dados, sendo produzida uma reflexão à luz dos aspectos teóricos do campo, na busca da compreensão a respeito da representação sobre a deficiência no serviço. As principais temáticas identificadas foram: concepção de deficiência; saúde da pessoa com deficiência; o papel da atenção primária perante esta população; práticas no cotidiano do serviço, e o trabalho da equipe de saúde.

\section{A pessoa com deficiência no contexto de um serviço de atenção primária à saúde: discurso e prática de profissionais}

A vida de um indivíduo com deficiência é entendida como muito difícil, estando em vigor um modelo tradicional de concepção da deficiência, chamado aqui de modelo da superação: ou seja, ele precisa ter habilidades extras e uma "força interna" superior para superar suas dificuldades e realizar suas ações no mundo, localizada essencialmente no próprio sujeito. 
O isolamento, a segregação, a não adaptação do meio social para a circulação da pessoa com deficiência na cidade, e os olhares de estranhamento aos quais são submetidas foram indicados como aspectos que agregam dificuldades no seu viver e na convivência com as pessoas. A pessoa com deficiência é vista como um peso para a família e para a sociedade, fazendo-se uma associação direta entre deficiência e condições mais graves de dependência e de falta de autonomia.

Marques (1998) aponta que as pessoas com deficiência, independentemente de suas potencialidades, estão amordaçadas em uma idéia totalizante de incapacidade, que diminui suas possibilidades de realização no mundo (sejam materiais, afetivas, educacionais, políticas etc.). Está presente o estigma (Goffman, 1988), ou seja, a pessoa é reduzida à condição dita negativa, na sua relação com os outros e com os diferentes contextos.

No relato de um dos profissionais entrevistados, ao descrever uma criança com deficiência, fica clara a dificuldade de se considerarem as características positivas do sujeito: "mas ele fala..., mas ele é inteligente". Para Pereira (2007), a frequente justificativa de uma ação com um "mas", ou um "apesar de", mostra a idéia da deficiência como um veredicto irretocável de incapacidade, ou seja, tomada como mais abrangente no corpo e na vida do que a relacionada com a real limitação existente.

Verificam-se dificuldades em relação ao reconhecimento da deficiência, como no caso de idosos ou pessoas portadoras de doenças crônicas incapacitantes. As produções atuais no campo da antropologia e sociologia da deficiência incluem estas condições na categoria "deficiência", reiterando que o conceito deve ser expandido, uma vez que experiências de incapacidade fazem parte do modo de viver dessa população (Diniz, 2007).

Os "olhares diferentes" para a pessoa com deficiência evidenciam a negação da dignidade, como citado por Vaitsman (2002). É importante ressaltar que os preconceitos e os tabus sobre a deficiência também estão presentes no encontro profissional-usuário, pois tanto o técnico quanto o paciente têm suas concepções e valores, a partir de sua história individual e social. É possível perceber que o usuário, muitas vezes, não reconhece estes aspectos, e o profissional tem dificuldades em fazer uma mediação para que a temática possa ser abordada de maneira mais integrada à vida cotidiana.

Com relação à saúde da pessoa com deficiência, a questão do acesso foi identificada como principal necessidade dessa população, entendida de maneira bastante ampliada, incluindo: acesso às oportunidades, à circulação na cidade, aos serviços e recursos disponíveis.

$\mathrm{Na}$ unidade de saúde em que foi realizada a pesquisa, existem algumas dificuldades na acessibilidade urbana e arquitetônica. Os profissionais trazem esta preocupação, bem como a importância de mudanças na estrutura física do prédio e nos arredores do centro de saúde. Entretanto, é preciso ressaltar que, ao mesmo tempo em que existem as barreiras, há uma organização do serviço para possibilitar o atendimento a todos os usuários: sala específica para atendimento no andar térreo, criação do programa de atenção domiciliar e previsão de reforma do prédio.

Em seus discursos, os profissionais entrevistados consideram que a atenção primária deve estar voltada a não reduzir as necessidades dos sujeitos a processos fisiopatológicos, provendo uma assistência integral e incorporando as dimensões subjetivas das práticas (tanto dos usuários dos serviços como dos profissionais). Porém, ainda que sejam feitas estas considerações, a assistência à pessoa com deficiência é frequentemente associada aos serviços especializados (como grandes hospitais e centros de reabilitação), especialmente aqueles de natureza filantrópica.

Existem diferenças nos focos de atenção entre as categorias profissionais: os médicos voltam-se para o corpo e as doenças; os técnicos de enfermagem atentam-se para os cuidados com feridas, as orientações e o apoio aos familiares/cuidadores; e os agentes comunitários, para as questões sociais, informações, direitos e cidadania. Estas especificidades indicam complementaridade dos trabalhos e das ações no serviço, possibilitando um olhar e uma atuação mais integrada para as demandas e necessidades dos sujeitos atendidos.

$\mathrm{Na}$ atenção à saúde da pessoa com deficiência, os limites entre a atenção primária e outros serviços de saúde ficam evidenciados - e como, na realidade dos serviços, estas atribuições não estão claras, emergem situações de tensão na integração entre a rede existente. Na literatura, há algumas produções que apresentam tecnologias específicas para ações voltadas à saúde da pessoa com deficiência no contexto da atenção primária; como mencionado anteriormente, é muito discutido o modelo da 
Reabilitação Baseada na Comunidade como proposta assistencial para o campo, por autores como Oliver et al. (2004, 1999) e Almeida, Tissi e Oliver (2000).

Há aproximações possíveis destas produções com o campo da atenção primária em geral, como nas publicações de autores como Schraiber e Mendes-Gonçalves (2000): tem-se, como objeto, a concepção de saúde além da doença e do corpo biológico, com o horizonte da promoção da saúde, em abordagens baseadas na autonomia dos sujeitos.

Na prática da assistência do serviço estudado, os principais focos de atenção estão relacionados ao corpo e às patologias, especialmente as doenças crônicas. Nos registros em prontuários, prevalecem informações como: diagnósticos clínicos, cuidados com higiene, orientações sobre curativos e prescrição de medicações. São recorrentes também descrições sobre as situações de dependência observadas, especialmente para execução de atividades de vida diária e dificuldades de locomoção. O cotidiano dos usuários aparece nos registros e relatos por meio das situações de incapacidade, ou seja, daquilo que a pessoa com deficiência não é capaz de fazer de maneira autônoma ou independente.

Estes aspectos são de fundamental importância no cuidado da saúde, mas o usuário pouco aparece como sujeito capaz de agir no contexto em que vive. Vários exemplos surgiram na pesquisa: poucos registros de histórias da vida e do cotidiano dos usuários; a criança com deficiência não tem prontuário próprio; o agente comunitário de saúde nunca fez visita por demanda da pessoa com deficiência; os planos para construção de autonomia pouco aparecem, e não se explicitam os projetos de vida da pessoa.

O cuidado aos familiares é um importante foco de atenção no serviço, e são vários os registros sobre orientações dadas aos cuidadores acerca de procedimentos a serem realizados. Distanciando-se dos aspectos médicos e corporais, nota-se uma prática frequente de promoção de apoio familiar (por meio de escuta e acolhimento), mas que, muitas vezes, pode contribuir para a manutenção de uma situação de dependência da pessoa com deficiência.

$\mathrm{Na}$ análise dos prontuários, destacam-se as situações de deficiência com condições mais graves de incapacidade, e estes usuários acabam sendo atendidos na modalidade de atenção primária domiciliar; nos atendimentos realizados na unidade de saúde, registram-se os diagnósticos clínicos, no entanto, as condições mais leves de deficiências e incapacidades passam desapercebidas.

As pessoas com deficiência mais independentes procuram o centro de saúde com demandas bastante específicas, como busca por relatórios para o Instituto Nacional do Seguro Social (INSS) ou para a isenção tarifária no transporte público; não emergem demandas gerais de saúde ou outras questões ligadas à deficiência na assistência prestada. Também não existem ações específicas voltadas a esta população, havendo pouca participação desta em grupos educativos, reflexivos e terapêuticos ou em outras atividades comunitárias desenvolvidas; os encaminhamentos realizados são para outras especialidades médicas e serviços de reabilitação, não havendo registros de orientação sobre equipamentos sociais ou outros espaços de inclusão, ainda que o serviço disponha de um guia que inclui vários locais de lazer, educação e esporte.

O acesso aos serviços públicos especializados continua difícil: há registros, em todos os prontuários estudados, de longa espera para atendimento em algumas especialidades, além de impossibilidades de seguimento com as especialidades indicadas (falta de transporte adaptado, não compreensão dos resultados esperados por pacientes e familiares, falta de recursos financeiros).

No caso apresentado na Tabela 1, é possível verificar todas as questões discutidas: há registro em prontuário de alguns comentários do usuário - como preocupações, gostos, relações familiares e projetos - mas estes não são transformados em objetos específicos de trabalho pela equipe de saúde. Além da atenção primária prestada na unidade básica de saúde e no domicílio, a paciente foi encaminhada para serviço especializado de nível secundário e para serviço de maior complexidade assistencial, mas, no seu caso, não há registro em prontuário de encaminhamento para equipamentos sociais; quanto à rede social, há menção a benefício recebido da igreja.

Pode-se dizer que, nas práticas desenvolvidas no serviço estudado, há uma relação mais direta com o modelo médico, voltado ao corpo e ao restauro das condições orgânicas (Ghirardi, 1999). Este modelo concebe a deficiência como uma anormalidade e, mais do que isso, uma "tragédia pessoal" (Diniz, 2007), em que a experiência da deficiência não é resultado apenas das lesões corporais e orgânicas, 
mas do ambiente social hostil à diversidade. Predomina também a concepção de que as pessoas com deficiência devem ser acompanhadas somente por serviços de saúde especializados.

Porém, como já mencionado por Almeida, Tissi e Oliver (2000), as principais demandas e necessidades das pessoas com deficiência estão nos complexos processos sociais que envolvem a questão, para as quais o modelo médico é bastante insuficiente. No serviço em questão, os aspectos relacionados ao paradigma social (Ghirardi, 1999) - como participação social, direitos, cidadania e construção de projetos de vida - estão presentes predominantemente no discurso.

Fica evidente uma tensão entre prática e discurso: a fala dos profissionais sobre a abordagem das demandas e necessidades das pessoas com deficiência é ampla, englobando os aspectos relacionais e socioculturais da deficiência; entretanto, nas ações, o trabalho está voltado, sobretudo, para: o corpo, as incapacidades na vida cotidiana e os aspectos orgânicos da deficiência, ainda que seja um serviço-escola.

\section{Algumas considerações finais}

Percebe-se que o panorama de desassistência existente antes da criação do SUS se mantém. No âmbito das práticas e ações, prevalecem as instituições filantrópicas como principais referências de algum tipo de cuidado às pessoas com deficiência, assim como mencionado por Almeida, Tissi e Oliver (2000). O acesso aos serviços continua difícil, e os relatos de experiências negativas com os programas e recursos existentes no sistema público são constantes. A família e uma pequena rede de apoio agregada são as principais fontes de cuidados às pessoas com deficiência, sendo fundamental destacar que não existem políticas ou ações mais amplas voltadas para o apoio ao familiar e a esta rede.

Os profissionais e o serviço de saúde atuam em um determinado contexto, com seus valores, finalidades e práticas; a condição de ter deficiência é permeada por estigmas, conflitos e desigualdades. Incorporados nos serviços, nas políticas e na sociedade, discursos e práticas complementam-se para o direcionamento do trabalho e dos cuidados em saúde. Pode-se concluir que a representação dos profissionais de saúde acerca do fenômeno deficiência/reabilitação está vinculada ao paradigma que operam, em um modelo mais médico ou social, conforme trazido por Ghirardi (1999).

No serviço estudado, é possível relacionar a tensão discurso-prática com os diferentes modelos de compreensão e ação sobre a deficiência. Eles coexistem, assim como os diferentes valores sobre a deficiência; o modelo médico é dominante no plano das ações, porém, a partir dos discursos são abertas novas possibilidades de intervenção, com vistas à integralidade e à participação, pertinentes ao campo da atenção primária.

As contradições são fundamentais, pois apenas desta maneira são produzidas as transformações. É no enfrentamento de diferenças entre discursos e práticas que se constrói um contexto instaurador de necessidades, tanto para os usuários (na relação com suas demandas e problemas), quanto para os profissionais (na relação com seu objeto de trabalho). Somente assim é que são criadas possibilidades de reinvenções e transformações dos sujeitos, dos serviços e das ações.

O conceito de representação adotado - discurso e prática como realidades complementares, produzidos por uma construção dialética e articulados com o contexto social e cultural (Magnani, 1986) - possibilitou a apreensão da complexa configuração que existe entre o plano do falado e do feito.

No campo da saúde da pessoa com deficiência, é na relação do sujeito com seu meio sociocultural que se constata a existência das grandes barreiras a serem enfrentadas. Reitera-se a importância do papel da atenção primária no cuidado integral à saúde destas pessoas; no entanto, este ainda constitui um desafio, exigindo reflexão sobre as práticas atuais e sobre as possibilidades de construções futuras.

A partir deste estudo, fica premente o surgimento de outros trabalhos que tomem como objeto as demandas e necessidades de saúde das pessoas com deficiência e as tecnologias de ação em atenção primária para esta população, contribuindo para a implementação de práticas baseadas na integralidade do cuidado. 


\section{Colaboradores}

A autora Marilia Bense Othero participou de todas as etapas de elaboração do artigo: revisão bibliográfica, elaboração, discussões e revisão do texto. A autora Ana Silvia Whitaker Dalmaso participou das discussões e da revisão do texto, uma vez que foi orientadora da primeira autora na elaboração da pesquisa e do artigo.

\section{Referências}

ALMEIDA, M.C.; TISSI, M.C.; OLIVER, F.C. Deficiências e atenção primária em saúde: do conhecimento à invenção. Rev. Ter. Ocup. Univ. São Paulo, v.11, n.1, p.33-42, 2000.

BRASIL. Ministério da Saúde. Secretaria de Atenção à Saúde. Departamento de Ações Programáticas Estratégicas. Manual de legislação em saúde da pessoa com deficiência. 2.ed. Brasília: Editora do Ministério da Saúde, 2006.

Ministério da Saúde. Secretaria de Assistência à Saúde. Coordenação de Atenção a Grupos Especiais. Programa de Atenção à Saúde da Pessoa Portadora de Deficiência. Atenção à pessoa portadora de deficiência no Sistema Único de Saúde: planejamento e organização de serviços. Brasília, DF, 1993.

DINIZ, D. O que é deficiência. São Paulo: Brasiliense, 2007.

GHIRARDI, M.I.G. Representações da deficiência e práticas de reabilitação: uma análise do discurso técnico. 1999. Tese (Doutorado em Psicologia) - Instituto de Psicologia, Universidade de São Paulo, São Paulo. 1999.

GOFFMAN, E. Estigma: notas sobre a manipulação da identidade deteriorada. Trad. Márcia Bandeira de Mello Leite Nunes. 4.ed. Rio de Janeiro: LTC, 1988.

MAGNANI, J.G.C. Discurso e representação, ou de como os Baloma de Kiriwina podem reencarnar-se nas atuais pesquisas. In: DURHAM, E.R. et al. A aventura antropológica: teoria e pesquisa. Org. por Ruth C. L. Cardoso. Rio de Janeiro: Paz e Terra, 1986. p.127-40.

MALINOWSKI, B. Baloma: los espiritus de los muertos em las islãs Trobriand. In: Magia, ciencia, religión. Barcelona: Ariel, 1974.

MARQUES, C.A. Implicações políticas da institucionalização da deficiência. Educ. Soc., v.19, n.62, p.105-22, 1998.

OLIVER, F.C. et al. Participação e exercício de direitos de pessoas com deficiência: análise de um grupo de convivência em uma experiência comunitária. Interface Comunic., Saude, Educ., v.8, n.15, p.275-88, 2004.

OLIVER, F.C. et al. Reabilitação baseada na comunidade: discutindo estratégias de ação no contexto sociocultural. Rev. Ter. Ocup. Univ. São Paulo, v.10, n.1, p.1-10, 1999.

PEREIRA, R. Deficiência e autodeterminação humana: compaixão e insensibilidade no caso Vincent Humbert. Hist., Cienc., Saude - Manguinhos, v.14, n.1, p.119-34, 2007.

PREFEITURA DE SÃO PAULO. Aspectos demográficos. Subprefeitura do Butantã. Disponível em: <http://portal.prefeitura.sp.gov.br/subprefeituras/spbt/dados/0001/ portal/subprefeituras/spbt/dados/aspectos_demograficos/0001>. Acesso em: 24 ago. 2008.

ROCHA, E.F. Deficiência e reabilitação: questões histórias e epistemológicas. In: (Org.). Reabilitação de pessoas com deficiência: a intervenção em discussão. São Paulo: Roca, 2006. p.9-60. 
SCHRAIBER, L.B.; MENDES-GONÇALVES, R.B. Necessidades de saúde e atenção primária. In: SCRAIBER, L.B.; NEMES, M.I.B.; MENDES-GONÇALVES, R.B. (Orgs.). Saúde do adulto: programas e ações na unidade básica. 2.ed. São Paulo: Hucitec, 2000. p.29-47.

VAITSMAN, J. Desigualdades sociais e duas formas de particularismo na sociedade brasileira. Cad. Saude Publica, v.18, supl., p.37-46, 2002. 
$\mathrm{Na}$ atenção primária, as ações voltadas à saúde da pessoa com deficiência devem visar à promoção da interação social, à construção de direitos e à transformação do lugar da deficiência. O objetivo desta pesquisa foi compreender qual é a representação de profissionais de um centro de saúde do município de São Paulo sobre a deficiência e o papel da atenção primária no seu cuidado. Foi utilizada metodologia qualitativa baseada em entrevistas com profissionais e análise de prontuários. Identificou-se que os profissionais avaliam a vida da pessoa com deficiência como difícil, evidenciando-se situações de dependência. Os principais focos da atenção estão no corpo, nos cuidadores e nas condições de moradia; direitos e participação social têm menor destaque. A família e pequena rede social são referências para a organização do cuidado. Com este estudo, busca-se ampliar a reflexão sobre práticas em saúde, contribuindo para a implementação daquelas baseadas na integralidade do cuidado.

Palavras-chave: Pessoas com deficiência. Cuidados primários de saúde. Pesquisa sobre serviços de saúde. Prática profissional. Assistência integral à saúde.

\section{Disabled people in primary healthcare: professionals' discourse and practice in a healthcare teaching center}

In primary healthcare, actions for disabled people's healthcare should aim to promote social interaction, construct rights and transform the position of disablement. The objective of this study was to understand the representations of professionals at a healthcare center in the municipality of São Paulo regarding disablement and the role of the primary care for this. Qualitative methodology was used, based on interviews with professionals and analysis of patient records. It was found that the professionals' assessment was that the disabled patients' lives were difficult, thus showing situations of dependence. The main focus of care was on the body, on caregivers and on housing conditions; rights and social participation had less emphasis. The family and the small social network were the reference points for organizing care. Through this study, it is sought to expand the reflections on healthcare practices, thereby contributing towards implementing practices based on comprehensive care.

Key words: Disabled people. Primary healthcare. Health service research. Professional practice. Comprehensive healthcare.

\section{Personas con deficiencia en la atención primaria: discurso y práctica de profesionales en un centro de salud-escuela}

En la atención primaria las acciones dirigidas a la salud de la persona con deficiencia deben visar la promoción de la interacción social, la construcción de derechos y la transformación del lugar de la deficiencia. El objetivo de esta pesquisa ha sido comprender cual es la representación de profesionales de un centro de salud del municipio de São Paulo, Brasil, sobre la deficiencia y el papel de la atención primaria en su cuidado. Se utilizó metodología cualitativa basada en entrevistas con profesionales evaluan la vida de la persona con deficiencia como difícil, evidenciándose situaciones de dependencia. Los principales focos de la atención están en el cuerpo, en los cuidadores y en las condiciones de vivienda; derechos y participación social tienen menor destaque. La familia y la pequeña red social son referencias para la organización del cuidado. Con este estudio se trata de ampliar la reflexión sobre prácticas en salud, para la implementación de las que se basan en la integralidad del cuidado.

Palabras clave: Pesquisa sobre servicios de salud. Práctica profesional. Asistencia integral a la salud. 